\title{
MIS COMIENZOS EN LAPAROSCOPIA
}

\author{
Edvardo Sánchez de Badajoz
}

Unidad Docente de Urología. Departamento de Cirugía. Facultad de Medicina. Universidad de Málaga. Málaga. España.

Resumen.- OBJETIVO: El autor describe algo de sus comienzos en laparoscopia, de dónde le vinieron algunas de sus ideas, el porqué y los medios con que contó al empezar. Cuenta brevemente su aportación a la Urología, que puede servir de modelo para los que están empezando.

MÉTODOS: Desde que el autor era un adolescente, su padre, que era urólogo, supo transmitirle el entusiasmo por su trabajo; se sentía atraído por el instrumental desde el litotritor de Freyer hasta el resector de Iglesias. También se interesó especialmente por los libros de urología antiguos. Le causaba especial admiración ver operar en directo a don José María Gil Vernet y cuenta que fue el primer urólogo al que le vio usar un laparoscopio para el diagnóstico del testículo abdominal. En 1985, mientras estaba en la universidad de Oxford, se le ocurrió diseñar un sistema de balón para disecar el espacio retropúbico, que al cabo de los años daría lugar a la cirugía extraperitoneal. Al año siguiente tras leer el manual de laparoscopia de Semm, describió la varicocelectomía laparoscópica. Entre sus trabajos destaca el haber descrito en 1993 por primera vez la técnica de la cistectomía radical con conducto ileal laparoscópico. En 1997 ideó un sistema robótico, mediante brazo maestro esclavo, concebido inicialmente para la resección transuretral.

RESULTADOS/ CONCLUSIONES: Considera que una buena idea es algo valiosísimo, que no tiene precio, porque a todo aquel que tenga ambiciones y la consiga, le permitirá salir de la mediocridad.

Palabras clave: Sánchez de Badajoz. Laparoscopia. Varicocele. Cistectomía. Cistoplastia. Balón. Robot. Historia de la Urología.

Summary.- OBJECTIVES: The author describes his first steps in laparoscopic surgery and the sources of some of his ideas. He thanks his father's influence and the technical stimuli that catalysed his scientific curiosity. For the benefit of young urologists at the beginning of their careers he shows how the frustrations of working with the early instruments became the vital challenges that inspired creative solutions.

METHODS: His urological surgeon father inspired in his young son a passion for his calling. He developed an immediate and compelling interest in the shape and function of urological instruments like, for example, Freyer's lithotripter and the Iglesias resectoscope. Books of urological history and the works of pioneer urologists fascinated him. Watching José María Gil Vernet operate particularly impressed him and he says that Gil Vernet 
was the first urologist he saw using a laparoscope to diagnose an abdominal testicle. While working in an Oxford University hospital in 1985, he designed a baIloon device to dissect the retropubic space. This procedure was the precursor of what several years later became extraperitoneal surgery. The following year, he read the manual of Semm's laparoscopy and later described a laparoscopic varicocelectomy. In 1993, he published the first description of a laparoscopic radical cystectomy and ileal conduit. In 1997, he adapted a surgical robotic system with a master-slave arm to carry out firstly a transurethral resection.

RESULTS/CONCLUSIONS: He says that a good idea is beyond price because it helps the inspired individual to make true a long-held ambition and achieve the signal success that lifts him out of the mud of mediocrity.

Keywords: Sanchez de Badajoz. Laparoscopy. Varicocele. Cystectomy. Cystoplasty. Balloon. Robot. History of Urology.

\section{INTRODUCCIÓN}

Ante todo he de decir que es un honor que el Dr. Mariano Pérez Albacete me pida que escriba sobre cómo fueron mis comienzos en la laparoscopia, de dónde me vino la idea, el porqué y de los medios con que conté al empezar, para que la gente joven vea un modelo de trabajo de cómo se empieza una experiencia nueva. Sin embargo mi querido amigo Mariano, además de ser un poco exagerado, me pone en un serio aprieto porque, aunque uno tiene ya algo escrito, la realidad es que salvo algún que otro editorial más o menos reivindicativo, nunca he contado nada de mi vida personal. Con mucho gusto pero no sin esfuerzo, por primera vez me dedico a hablar de mí y aunque siempre he oído que debe evitarse el empleo del "yo", en este caso voy a desoír el consejo y voy a contar algo de cómo se desarrolló mi carrera, o lo que es igual, mi humilde aportación a la Urología y a la laparoscopia.

\section{MATERIAL Y MÉTODOS}

Mi padre era urólogo y desde que yo era un adolescente supo transmitirme el entusiasmo por su trabajo, hasta el extremo de que desde casi mi infancia yo ya conocía gran parte del instrumental, ya que mi maestro heredó toda una colección de instrumentos en gran parte decimonónicos y que todavía conservo como reliquias. Entre los muchos que tengo, probablemente mi preferido es un litotritor "ciego" de Freyer, que él manejaba con gran maestría. Sin embargo, desde que yo era un niño mi padre ya tenía un resector modelo Nesbit, que a mí me causaba sensación, pero que más tarde cambió por el de Iglesias, en el que se sustituyó el muelle en espiral por un fleje metálico y que fue el que usó toda su vida.

También casi desde mi adolescencia mi maestro tenía un bisturí eléctrico modelo Birtcher de lámparas, y que todavía conservo, con una precisión que nunca consiguieron los modelos de transistores y cuya calidad de corte no han igualado hasta los más recientes. Bisturí que le recomendó don Luís Cifuentes con quien mi padre tenía una estrecha amistad.

Además mi padre heredó una gran biblioteca de Urología, con no pocos libros de los primeros años del siglo, entre los que me apasionaban especialmente los de Joaquín Albarrán, por sus maravillosos dibujos y por su metodología impecable francesa, curiosamente como todos los libros franceses con los índices al final. Mi pasión por estos libros llegaba hasta tal extremo, de que cuando yo estaba a mitad de la carrera, en vez de dedicarme a estudiar ya sea psiquiatría o pediatría, llegué a saberme el maravilloso libro de "Técnica quirúrgica de Couvelaire" casi de memoria. Sin embargo, de todos los libros que por esos años leí hubo uno que me impactó y fue una monografía escrita por José Iglesias de la Torre en la Habana, en 1948, en donde hacía la descripción más magistral que he encontrado de una prostatectomía transuretral, hecha con las ópticas de entonces

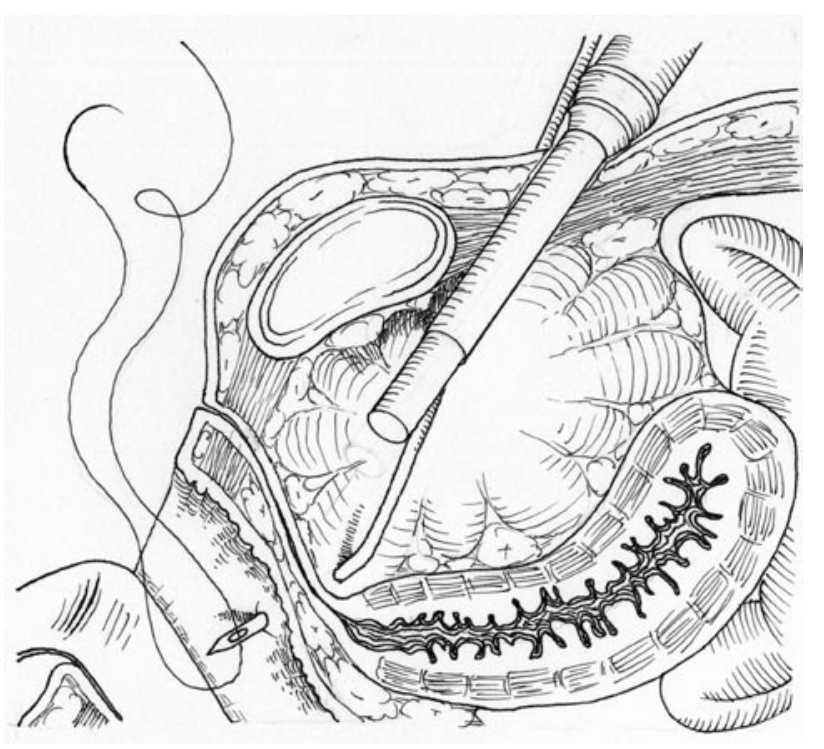

FIGURA 1. Una vez disecado el espacio retropúbico mediante un balón, se está realizando una uretropexia. 
y cuando todavía el endoscopio tenía una bombillita en la punta, que era la pesadilla del cirujano, ya que se fundía en el momento más inoportuno.

No puedo dejar de mencionar el tremendo impacto que me producía ver operar en directo a don José María Gil Vernet, con esa elegancia, esa magia y esa infalibilidad que me parecían sobrehumanas y que estoy convencido de que nadie ha conseguido superar. Y es más, don José María, curiosamente utilizó el laparoscopio antes que todos nosotros, ya que fue quien realmente popularizó su uso para el diagnóstico del testículo abdominal, previo al autotrasplante. Técnica que aprendí de él.

Tras varios años de formación como urólogo pensé que había llegado la hora de ir al extranjero. El primer año, en 1985, estuve en la Universidad de Oxford donde tuve la ocasión de hacer por un lado gran parte de mi tesis doctoral, consistente en trasplante pancreático experimental, que realizaba en el Hospital John Radcliffe, en el departamento de Cirugía, que dirigía el Profesor Morris. Trabajo que simultaneaba con mi asistencia al Hospital Churchil, situado muy cerca, donde estaba el Departamento de Urología y que dirigía el Dr. Joseph Smith, que fue presidente de la Asociación Británica de Urología. La realidad es que a pesar de mi trabajo hospitalario, yo disponía de mucho tiempo, que en gran parte pasaba en la inmensa biblioteca del Hospital llamada "Cairns" en honor de su benefactor. Yo tenía una visión muy clara de lo que quería, la razón de mi estancia en la biblioteca no era adquirir una cultura urológica o quirúrgica, que en parte ya tenía, ni siquiera ampliar mi formación en términos genera- les, sino que era una misión concreta y precisa. Se trataba de buscar una idea específica, que sencillamente me permitiera describir una técnica quirúrgica original y novedosa. Por esas fechas fue cuando me di cuenta de que la inspiración no es algo caído del cielo, sino que hay que buscarla, a veces con un tremendo esfuerzo, y también entonces comprendí que para perseguir una idea con tanta energía, hay que tener poderosas razones, que incluso rayen la obsesión y que después comentaré.

Después de semanas de indagaciones se me ocurrió que si yo introducía una sonda de balón en el espacio retropúbico y la inflaba hasta unos 300 mililitros, podría despegar el peritoneo y crear una gran cavidad donde poder trabajar y que unos diez años más tarde se convertiría en la cirugía retroperitoneal (Figura 1). Como a menudo ocurre, el artículo cursó sin pena ni gloria, pero unos diez años más tarde tuve la satisfacción de comprobar que fue la primera vez que se hizo una cirugía endoscópica extraperitoneal mediante sistema de balón $(1,2)$.

Como dato anecdótico, hace pocos años, con motivo de un pleito entre dos casas comerciales, tuve el honor de que me llamaran de un juzgado de San José de California, donde acudí como experto. El primer día me citaron en una habitación donde había una mesa alargada donde cabrían unas 25 personas, me sentaron en uno de los extremos, fren-

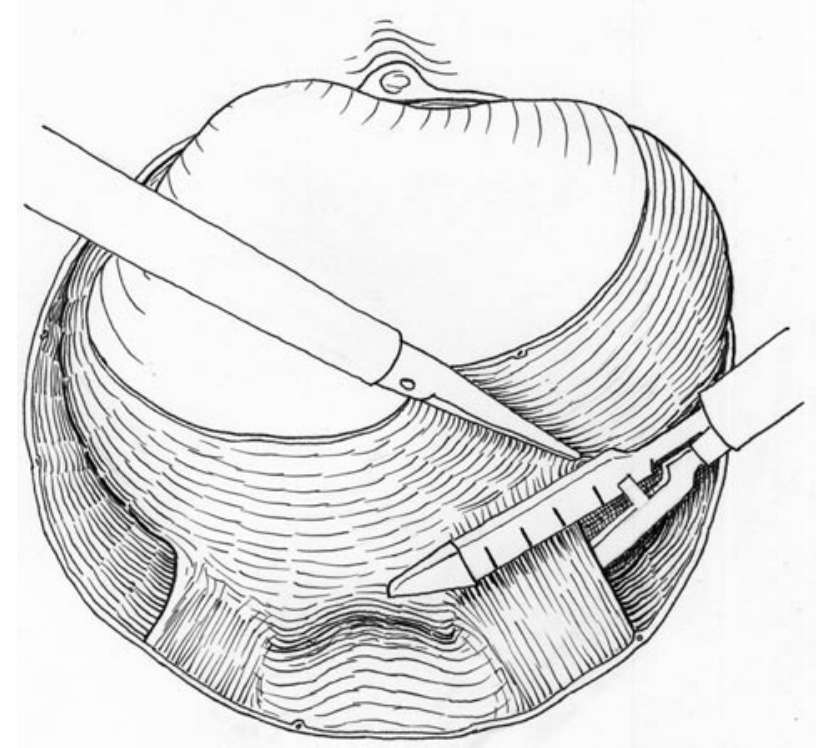

FIGURA 3. Sección de los pedículos en la cistectomía
laparoscópica.

FIGURA 3. Sección de los pedículos en la cistectomía
laparoscópica.
FIGURA 2. Varicocelectomía laparoscópica mediante visión directa cuando todavía no había cámaras operatorias.

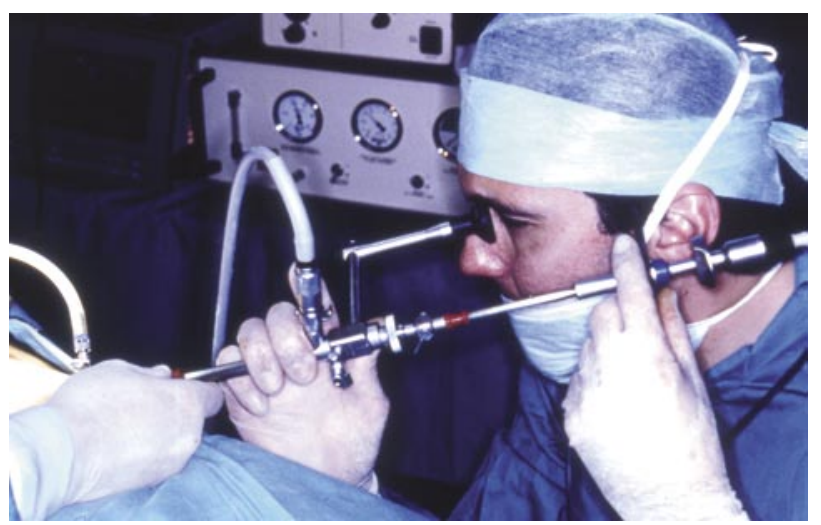


te a mí pusieron una videocámara de las grandes y durante unas dos horas me estuvieron preguntando cosas sobre el balón. Al día siguiente fue el juicio y tras el consabido juramento, me estuvieron interrogando durante cuatro horas sobre el dichoso balón. La realidad es que aparte de colmar mi vanidad, no saqué ningún beneficio de aquella aventura.

En 1986 pasé una temporada en el Departamento de Urología del "London Hospital", que por esas fechas dirigía el Profesor Blandy. La lectura de sus libros me había fascinado, en especial los dibujos esquemáticos hechos por el propio autor. Además, sus textos tenían muy a menudo un fino y sutil sentido del humor que a veces era genial; humor inglés en definitiva. Una vez allí me llamó la atención que sus historias clínicas y hojas operatorias eran cortas y estaban llenas de esquemas y dibujos, los mismos que en sus libros. También me chocó la claridad y sobre todo la brevedad de los informes médicos, que eran el polo opuesto a lo que yo había visto en España,

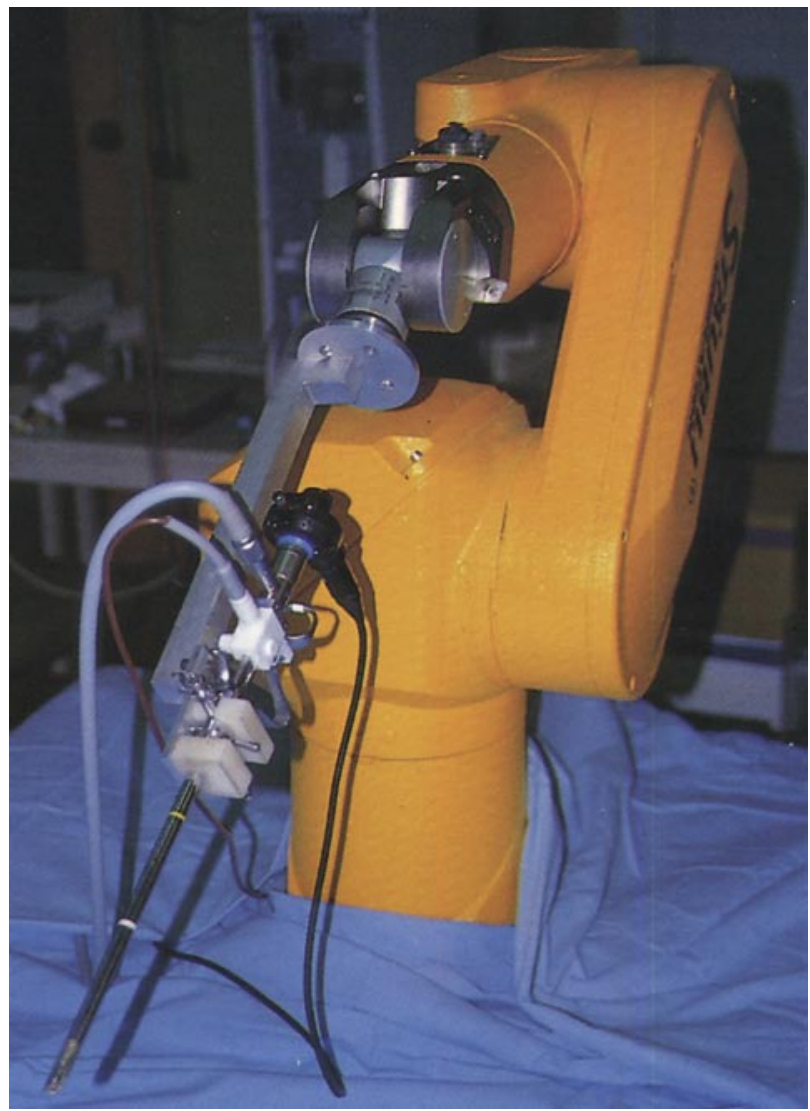

FIGURA 4. Robot para la cirugía endoscópica mediante control remoto. donde la mayoría de las veces los informes eran monótonos, ambiguos, aburridos e interminables. Fue entonces cuando aprendí que hay que "respetar el tiempo del lector" y que si uno quiere que la gente lea sus escritos hay que ser breve.

Mi filosofía ese año era la misma que la del año anterior en Oxford, es decir, yo no estaba allí de vacaciones y al final de mi estancia, tenía que venirme por lo menos con una publicación que se saliera de lo habitual. Entre todos los muchos textos que consulté, cayó en mis manos un libro que me pareció mágico, se trataba del manual de laparoscopia de Semm, que para el que no lo conozca, era un ginecólogo creador de la cirugía laparoscópica actual, donde por primera vez se describían las técnicas de endosutura y endoligadura. Tras su lectura, todo mi afán era buscar una aplicación a la Urología. La verdad es que no me fue fácil, ya que me pasé días o quizá semanas dándole vueltas, como he dicho antes, de forma casi obsesiva. No se me olvidará, una noche, una vez que me había acostado, me vino la inspiración "divina", salté de la cama e hice un dibujo donde representé la varicocelectomía laparoscópica. Naturalmente no se lo dije a nadie, porque soy un poco paranoico con eso de que me roben los"inventos", aunque probablemente no me habrían hecho caso (Figura 2). Y cuando volví a casa, operé una serie de casos con buenos resultados $(3,4)$.

Hace diez años ya habíamos realizado bastante laparoscopia, incluyendo una serie de cistectomías (Figura 3) e incluso varias enterocistoplastias $(5,6,7,8,9)$. Pues bien, con esa inquietud rayando lo místico que siempre me ha "atormentado", pensé que esté tipo de cirugía, tal y como estaba concebida, había tocado fondo y que tenía que modificarse. Se me ocurrió hacer una búsqueda bibliográfica con las palabras "cirugía y robótica" y encontré tan sólo una docena de artículos. Ese nuevo campo me pareció fascinante, me sorprendió lo poco que se había hecho en esa fecha, lo que quería decir que era una materia con enormes posibilidades y donde habría mucho que aportar $(10,11)$. A través de un amigo conseguí contactar con uno de los profesores de robótica de nuestra universidad y le propuse realizar un artilugio que permitiera realizar la resección transuretral mediante control remoto, es decir una resección mediante "brazo maestro esclavo", término que antes nadie había utilizado en Urología y que ahora está tan de moda. Pocos meses después el robot estaba fabricado y funcionando (Figura 4). Cerca de un año más tarde, con la experiencia acumulada, también empezamos a trabajar dentro de la laparoscopia. Fruto de dicha colaboración con los ingenieros han sido una serie de artículos e incluso varias patentes $(12,13,14)$. 


\section{RESULTADOS/DISCUSIÓN}

Hay un refrán que dice que en los EEUU, teniendo una buena idea y un teléfono, uno se hace millonario. Yo no vivo precisamente en el país de las oportunidades, pero estoy totalmente de acuerdo en que una buena idea es algo valiosísimo que no tiene precio. Y para llevarla a cabo hay que tener un gran entusiasmo y motivación, ya que la creatividad no es fácil y mucho menos en este campo.

Se me pide que diga cuál ha sido el estímulo para tener esa serie de ideas. La realidad es que motivos sí que los hay como para casi todo, pero no me resulta fácil contarlos. En mi caso concreto, el hecho de que mi padre fuera urólogo suponía muchas ventajas. Sin embargo, como todos sabemos, ser hijo de alguien es muy difícil, porque los méritos personales inevitablemente son menos valorados, y eso me resultaba especialmente penoso, lo que hacía que yo ambicionara salir de la mediocridad, y qué mejor que publicando, y si es posible, ideas interesantes.

Naturalmente que al poco tiempo ésa dejó de ser la razón de mi inquietud creadora. Sin embargo, esa ambición, ese desasosiego, habían germinado y se habían convertido en algo habitual en mí, y es lo que ha hecho que a lo largo de los años yo haya seguido luchando, tratando de ser innovador, hasta el extremo de que la mayor satisfacción que me produce esta profesión, por encima de todo, es ver un artículo publicado, y no digamos si encima le gusta a la gente.

\section{BIBLIOGRAFÍA Y LECTURAS RECOMENDADAS (*lectura de interés $y^{* *}$ lectura fundamental)}

1. 1. SÁNCHEZ DE BADAJOZ, E.; DÍAZ RAMIREZ, F.; MARÍN MARTÍN, J.: “Anclaje cervical endoscópico. Nuevo tratamiento para la incontinencia de esfuerzo". Arch. Esp. Urol., 41: 127, 1988.
2. SÁNCHEZ DE BADAJOZ, E.; DÍAZ RAMIREZ, F.; VARA THORBECK, C.: "Stress incontinence: A new endoscopic approach". Urology, 36: 403, 1990.

**3. SÁNCHEZ DE BADAJOZ, E.; DÍAZ RAMÍREZ, F.; MARÍN MARTÍN, J.: "Tratamiento endoscópico del varicocele". Arch. Esp. Urol., 41: $15,1988$.

4. SÁNCHEZ DE BADAJOZ, E.; DÍAZ RAMIREZ, F.; VARA THORBECK, C.: "Endoscopic varicocelectomy”. J. Endourol., 4: 371, 1991.

5. SÁNCHEZ DE BADAJOZ, E.; GALLEGO PERALES, J.L.; RECHE ROSADO, A. y cols.: "Cistectomía radical y conducto ileal laparoscópico". Arch. Esp. Urol., 46: 621, 1993.

*6. SÁNCHEZ DE BADAJOZ, E.: "Laparoscopic cystectomy and ileal conduit: a case report". J. Endourol., 9: 59, 1995.

7. SÁNCHEZ DE BADAJOZ, E.; MATE HURTADO, A.; JIMÉNEZ GARRIDO, A. y cols.: "Cistoplastia laparoscópica". Arch. Esp. Urol., 46: 615, 1993.

8. SÁNCHEZ DE BADAJOZ, E.: "Laparoscopic assisted cystoplasty". J. Endourol., 9: 269, 1995.

9. SÁNCHEZ DE BADAJOZ, E.; GÓMEZ DE LA CRUZ, J.M.; JIMÉNEZ GARRIDO, A. y cols.: "Cirugía vasculorrenal endoscópicamente asistida". Arch. Esp. Urol., 48: 2, 1995.

10. SÁNCHEZ DE BADAJOZ. E.: "El paso siguiente". Arch. Esp. Urol., 50: 1031, 1997.

11. SÁNCHEZ DE BADAJOZ. E.: "Robótica y Urología". Arch. Esp. Urol., 51: 215, 1998.

12. SÁNCHEZ DE BADAJOZ, E.; JIMÉNEZ GARRIDO, A.; MUÑOZ FERNÁNDEZ, V.F. y cols.: "Resección transuretral mediante control remoto". Arch. Esp. Urol., 51: 445, 1998.

13. JIMÉNEZ GARRIDO, A.; SÁNCHEZ DE BADAJOZ, E.; MUÑOZ FERNÁNDEZ, V.F. y cols.: "Cistoscopia robotizada dirigida mediante la voz". Arch. Esp. Urol., 52: 374, 1999.

14. SÁNCHEZ DE BADAJOZ, E.; JIMÉNEZ GARRIDO, A.; GARCÍA VACAS, F. y cols.: "Nuevo brazo maestro para la resección transuretral mediante robot". Arch. Esp. Urol., 55: 1247, 2002. 